\section{- OPEN ACCESS}

\title{
Immune-mediated effects targeting hepatitis C virus in a syngeneic replicon cell transplantation mouse model
}

\author{
Sepideh Levander, ${ }_{1}^{1}$ Fredrik Holmström, ${ }^{1}$ Lars Frelin, ${ }^{1}$ Gustaf Ahlén, ${ }^{1}$ Daniel Rupp, ${ }^{2,3}$ \\ Gang Long, ${ }^{2,4}$ Ralf Bartenschlager, ${ }^{2,5,6}$ Matti Sällberg ${ }^{7,8}$
}

Additional material is published online only. To view please visit the journal online (http://dx.doi.org/10.1136/ gutjnl-2016-313579).

For numbered affiliations see end of article.

\section{Correspondence to}

Ralf Bartenschlager, Department of Infectious Diseases,

Molecular Virology, University of Heidelberg, D-69120

Heidelberg, Germany; ralf. bartenschlager@med.uniheidelberg.de and Professor Matti Sällberg, Department of Laboratory Medicine, Division of Clinical Microbiology, F68, Karolinska Institutet, Karolinska University Hospital Huddinge, S-141 86 Stockholm, Sweden; matti.sallberg@ki.se

$\mathrm{RB}$ and MS contributed equally.

Received 13 December 2016 Revised 27 April 2017 Accepted 20 May 2017 Published Online First 1 June 2017
Check for updates

To cite: Levander $S$, Holmström F, Frelin L, et al. Gut 2018:67:1525-1535.

\section{ABSTRACT \\ Objective $\mathrm{HCV}$ is characterised by its ability to} establish chronic infection in hepatocytes and to replicate in the presence of an inflammation. We mimicked this situation in vivo in immune-competent mice by syngeneic transplantation of HCV replicon-containing mouse hepatoma cells.

Design A total of 5 million $\mathrm{H}-2^{b}$ positive Hep56.1D cells, carrying a subgenomic genotype (gt) 2a replicon (HCV replicon cells) or stably expressing comparable levels of the HCV NS3/4A protease/helicase complex (NS3/4A hepatoma cells), were injected subcutaneously into syngeneic $\mathrm{H}-2^{b}$-restricted mice. Kinetics of tumour growth, HCV RNA replication levels and HCV-specific immune responses were monitored. For immune monitoring, new $\mathrm{H}-2^{\mathrm{b}}$-restricted cytotoxic T cell epitopes within the gt2a NS3/4A region were mapped. Immune mice were generated by DNA-based vaccination. Results HCV replicon and NS3/4A hepatoma cells generated solid tumours in vivo. Similar to what is seen in human HCV infection did HCV RNA replicate in the presence of inflammation. NS3/4A-specific CD8+ T cells seemed to transiently reduce HCV RNA levels. Both CD4+ and CD8+ T cells were required for protection against tumour growth. Vaccine-induced NS3/4A(gt2a)specific T cells protected against HCV replicon tumours in wild-type, but not in HCV NS3/4A(gt1a)-transgenic mice with dysfunctional HCV-specific T cells. Importantly, as in human HCV infection, HCV replicon cells neither primed nor boosted a strong NS3/4A-specific T cell response.

Conclusion Syngeneic transplantation of mouse HCV replicon cells into immune-competent animals mirrors many in vivo events in humans. This system is versatile and can be applied to any genetically modified $\mathrm{H}-2^{\mathrm{b}}$ restricted mouse strain.

\section{INTRODUCTION}

New direct-acting antivirals have recently become available that effectively cure chronic infections caused by the HCV. However, there is still no effective vaccine against HCV. One reason is the lack of simple and reliable small animal models in which vaccines can effectively be evaluated. A number of HCV vaccines have been taken towards clinical testing with varying results. ${ }^{1-8}$ One factor that can facilitate vaccine development is immune-competent small animal models that share features with the HCV infection of humans. Several useful,

\section{Significance of this study}

What is already known on this subject?

- There is no fully immune-competent small animal model for HCV.

- In humans, HCV most often escapes control by innate and adaptive immune responses, but many mechanistic details are unknown.

- For reasons that are not well understood, HCV infection triggers a poor immune response that in most cases is not protective.

What are the new findings?

- This is the first fully immune-competent small and versatile animal model allowing HCV RNA replication.

- Similar to the infection of humans, HCV RNA can replicate in the presence of a strong inflammatory response.

- Similar to the infection of humans, hepatoma cells carrying an HCV replicon prime only weak HCV-specific T cell responses.

- Syngeneic transplantation of HCV replicon cells can be rapidly applied to any gene-deficient or transgenic C57BL/6J-based mouse linaege.

How might it impact on clinical practice in the foreseeable future?

- This in vivo model may give unique insights into the HCV biology and importatly assist in vaccine development.

although limited, models have been developed, such as regular transgenic mice with hepatic expression of one or more HCV proteins. ${ }^{9-15}$ More recently, a model using immune-deficient STAT $1^{-/-}$mice with transgenic expression of human HCV entry factors has been developed. ${ }^{16}$ However, these models lack either HCV RNA replication or functional immunity. Thus, there is a need for models that allow the evaluation of potential $\mathrm{HCV}$ vaccine candidates with more challenging tests and therefore are better predictive of vaccine efficacy in humans. This has recently been highlighted by the observation that vaccine-induced $T$ cells have none or very transient effects in human HCV infections. ${ }^{4-7}$

We here used a previously developed HCV replicon adapted to murine Hep56.1D hepatoma cells ${ }^{17}$ 
in a syngeneic transplantation setting and show that this mouse model mimics important aspects of the HCV-specific immune response in infected humans.

\section{MATERIALS AND METHODS \\ Cell lines}

The murine H-2b-restricted Hep56.1D hepatoma cell line was obtained from CLS Cell Line Services. ${ }^{18}$ Hep56.1D cells were maintained in a humidified incubator at $37^{\circ} \mathrm{C}$ with $5 \% \mathrm{CO}_{2}$ in Dulbecoo's Modified Eagle's Medium (DMEM) supplemented with $10 \%$ fetal bovine serum (FBS), $100 \mathrm{U} / \mathrm{mL}$ penicillin and $100 \mu \mathrm{g} / \mathrm{mL}$ streptomycin, $1 \mathrm{mM}$ non-essential amino acids and 2 mM GlutaMAX-I (Sigma-Aldrich, St Louis, Missouri, USA). Hep56.1D cells containing a subgenomic HCV replicon of genotype 2a (isolate JFH-1) and designated Hep56-sgJFH-cl3 and Hep56-sgJFH-cl10 have been described previously. ${ }^{17}$ Cells were grown under selection with $750 \mu \mathrm{g} / \mathrm{mL}$ G418. To facilitate in vivo monitoring, $\mathrm{HCV}$ replicon cells were stably transfected with the pGL4.50 (luc2/cytomegalo virus early immidieate promoter (CMV)/Hygro) vector encoding the firefly luciferase gene (Promega, Madison, Wisconsin, USA). These cells were passaged in complete DMEM, containing in addition $200 \mu \mathrm{g} /$ $\mathrm{mL}$ hygromycin (Sigma-Aldrich). By using quantitative PCR we found that each HCV replicon-luciferase cell contained two chromosomally integrated copies of the luciferase gene. This allowed determination of the number of HCV replicon cells in tumour tissue. As a control Hep56.1D cells were stably transfected with a functional JFH-1 NS3/4A-encoding plasmid by using standard protocols (NS3/4A hepatoma cells). Cells were passaged in complete DMEM containing $1000 \mu \mathrm{g} / \mathrm{mL}$ G418 in a humidified incubator at $37^{\circ} \mathrm{C}$ and $5 \% \mathrm{CO}_{2}$. $\mathrm{HCV}$ replicon and NS3/4A hepatoma cell lines expressed similar levels of NS3 protein (figure 1).

\section{Used DNA constructs}

Detection and quantification of NS3 protein expression were performed as previously described. ${ }^{19}{ }^{20}$ A full-length codon-optimised (co) JFH-1 NS3/4A gene was generated synthetically (Retrogen, San Diego, California, USA) and inserted into the pVAX1 vector backbone (Invitrogen, Carlsbad, California, USA). The plasmid coNS3/4A-pVAX1 (GenBank accession number: AR820945.1; http:/www.nbci.nlm.nih.gov/ genbank) containing the full-length coNS3/4A gene of $\mathrm{HCV}$ genotype $1 \mathrm{a}$ has been described previously. ${ }^{21}$ The following DNA vaccines were used as negative controls: The chicken ovalbumin (OVA)-pCI plasmid ${ }^{22}$ expressing the secreted (s) product sOVA-C1 was kindly provided by Dr Andrew Lew (WEHI, Melbourne, Australia). The sOVA-C1 gene was inserted into the pVAX1 backbone. The co HBcAg-pVAX1 plasmid (GenBank accession number DI244925.1) has been described previously. ${ }^{23}$ Additional details have been given in the online supplementary materials.

\section{Animals}

Inbred female $\mathrm{C} 57 \mathrm{BL} / 6 \mathrm{~J}\left(\mathrm{H}-2^{\mathrm{b}}\right)$ mice were obtained from Charles River, Sulzfeld, Germany. CD $4^{-/-}\left(\mathrm{H}-2^{\mathrm{b}}\right)$ and $\mathrm{CD} 8^{-/-}\left(\mathrm{H}-2^{\mathrm{b}}\right)$ mice were obtained from the Jackson Laboratory (Bar Harbor, Maine, USA). Interferon-gamma receptor-2 (IFN- $\gamma \mathrm{R} 2)^{-/-}\left(\mathrm{H}-2^{\mathrm{b}}\right)$ mice were bred and maintained in-house at Karolinska Institutet, Division of Comparative Medicine, Clinical Research Center, Karolinska University Hospital, Huddinge, Sweden. Mice transgenic for full-length wild-type HCV NS3/4A of gt1a (NS3/4A-Tg)

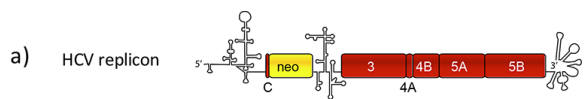

b)
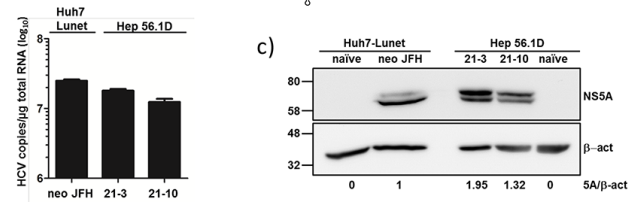

d)
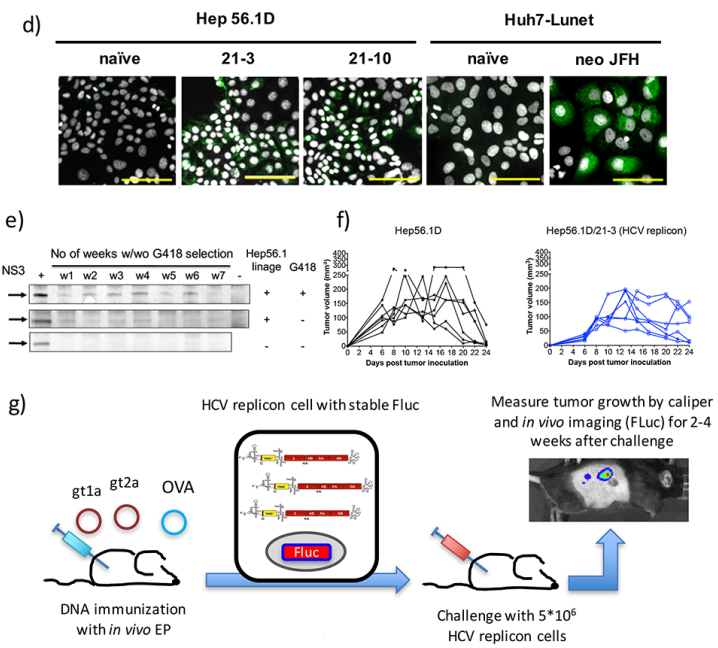

Figure 1 Schematic of the experimental approach and characterisation of Hep56.1D-derived replicon cells suitable for syngeneic transplantation. (A) Schematic of the subgenomic HCV gt2a (isolate JFH-1) replicon in Hep56.1D (C57BL/6J-derived) hepatoma cells. Non-coding RNA regions are displayed with their presumed secondary structures. HCV proteins NS3, 4A, 4B, 5A and 5B are indicated with brown boxes. The G418 resistance gene (neo) is given as yellow box; the N-terminal fusion with 16 codons of the HCV core (C) protein is indicated.17 (B) Quantification of HCV RNA in human (Huh7-Lunet-derived) and mouse (Hep56.1D-derived) replicon cells (clones 21-3 and 21-10) by using RT-qPCR. Values were normalised to glyceraldehyde 3-phosphate dehydrogenase (GAPDH) and quantified by using a HCV standard. (C) Quantification of NS5A in Huh7-derived and Hep56.1D-derived replicon cells. Equal protein amounts of each sample were loaded onto the gel. Beta-actin ( $\beta$-act) served as loading control. Ratios of NS5A and $\beta$-act-specific signals are given below the respective lanes. The ratio determined for Huh7-Lunet cells containing the selectable JFH-1 replicon was set to 1. (D) Immune fluorescence analysis of HCV NS3 (green) in Hep56.1D and Huh7-Lunet-derived cells. Naïve cells served as specificity control. Nuclei were stained with 4',6-diamidino-2-phenylindole (DAPI) (grey). Scale bar, 100 $\mu \mathrm{m}$. (E) Detection of HCV NS3 protein expression in Hep56-derived cells containing the subgenomic replicon (cell clone 21-3) passaged for 7 weeks in the presence or absence of G418 (upper and middle panel, respectively). NS3 was detected by immunoprecipitation and western blot analysis. A lysate of Huh7 cells transiently transfected with an NS3/4A construct was used as size marker and positive control (left lane). Parental Hep56.1D cells served as negative control (lower panel and right lane). (F) Groups of 6-7 wild-type C57BL/6J mice were inoculated subcutaneously with $5 \times 10^{6} \mathrm{HCV}$ replicon-containing cells or the parental hepatoma cells (Hep56.1D). Tumours were measured every second to third day after inoculation (ie, days $6,8,10,13,15,17,20,22$ and 24). The tumour volume of individual mice is shown. No statistically significant difference was found between mouse groups by comparing the area under the curve and analysis of variance. NS, not significant. (G) Experimental approach used for cell transplantation and in vivo analysis of tumour cells. Note that HCV replicon cells were also stably transfected with a luciferase reporter gene (Fluc). 
were previously described. ${ }^{24}$ Additional details have been given in the online supplementary materials.

\section{Peptides and proteins}

A total of 7520 -amino acids long peptides with 10 aa overlap covering the full-length NS3/4A-JFH-1 sequence were purchased from Sigma-Aldrich. Details have been given in the online supplementary materials.

\section{Immunisation protocols}

Mice (5-20 per group) were immunised intramuscularly in the tibialis cranialis (TC) muscle ${ }^{2526}$ one or two times with $0.5-50 \mu \mathrm{g}$ plasmid DNA as described in the online supplementary materials.

\section{In vivo challenge with HCV replicon and NS3/4A-expressing Hep56.1D cells and bioluminescence imaging}

In vivo challenge with HCV replicon cells or the NS3/4A hepatoma cells was done in naïve and immunised mice 2 weeks after the last immunisation using $5 \times 10^{6}$ tumour cells. The cells were washed, resuspended in $200 \mu \mathrm{L}$ phosphate buffered saline (PBS) and inoculated subcutaneously into the right flank of the mouse. The kinetics of tumour growth was determined by measuring the tumour volumes through the skin using a sliding calliper every second or third day. The volume was calculated by using the formula: $0.5 \times$ (tumour length $\times$ tumour diameter $\left.{ }^{2}\right) .{ }^{27} \mathrm{HCV}$ replicon cell tumours were also monitored for luciferase activity using the IVIS Spectrum in vivo imaging system (Xenogen IVIS Spectrum, Caliper Life Sciences, Hopkinton, Massachusetts, USA). To detect luciferase expression in vivo, mice were shaved and injected with $15 \mathrm{mg} / \mathrm{kg}$ body weight luciferin substrate (D-Luciferin, $\mathrm{K}^{+}$salt, PerkinElmer, Waltham, Massachusetts, USA) diluted in $200 \mu \mathrm{L}, 4 \mathrm{~min}$ prior to anaesthesia of the animals with isoflurane (IsoFlo, Abbott Laboratories, Berkshire, UK). Mice were analysed in the IVIS machine $11 \mathrm{~min}$ after the luciferin injection. Images and assessment of emitted light were analysed (Living Image Software V.4.2).

\section{Extraction of RNA and DNA and quantitative real-time PCR}

To allow for quantification of HCV RNA levels and to determine the total number of luciferase copies in tumour tissue or cells, purifications of RNA and DNA were performed. Details have been given in the online supplementary materials.

\section{Chromogenic in situ hybridisation of formalin-fixed, paraffin- embedded sections}

Chromogenic in situ hybridisation was performed using the ViewRNA ISH Tissue Assay Kit and ViewRNA Chromogenic Signal Amplification Kit provided by Affymetrix as described in the online supplementary materials.

\section{Detection of interferon-gamma (IFN $\gamma$ )-producing T cells by Enzyme-Linked ImmunoSpot (ELISpot)}

\section{Assay}

Splenocytes from each group of mice were pooled and tested for the presence of NS3/4A-specific T cells. Production of IFN $\gamma$ was determined by using a commercially available ELISpot assay (Mabtech, Nacka Strand, Sweden) exactly as described previously $^{28}$ using splenocytes from groups of immunised and/or tumour cell-challenged mice. Details are given in the online supplementary materials.

\section{Quantification of HCV NS3 gt2a-specific CD8+ T cells}

The frequency of NS3-specific CD8 + T cells was analysed by ex vivo staining of splenocytes using the recombinant soluble dimeric mouse $\mathrm{H}-2 \mathrm{D}(\mathrm{b}): \mathrm{Ig}$ fusion protein (BD Biosciences, San Jose, California, USA) as described previously. ${ }^{21} 29$ In brief, $1 \times 10^{6}$ spleen cells were resuspended in PBS/1\% FBS (FACS buffer) and incubated with Fc-blocking antibodies. Cells were then washed and incubated for 90 min with $\mathrm{H}-2 \mathrm{D}(\mathrm{b})$ :Ig preloaded with a NS3-derived major histocompatibility complex (MHC) I peptide (eg, NS3 cytotoxic T lymphocyte (CTL) epitope with the amino acid sequence APPPSWDAM, H-2 ${ }^{\mathrm{b}}$ ). Thereafter, cells were washed and incubated for $30 \mathrm{~min}$ with a PE-conjugated rat antimouse IgG1 antibody. Cells were then washed and incubated for $30 \mathrm{~min}$ with APC-conjugated rat antimouse CD19 and FITC-conjugated rat antimouse CD8 antibodies. A total of 150000 events from each sample were acquired on a FACSVerse flow cytometer (BD Biosciences) and analysed using the FlowJo V.9.2 software (Ashland, Oregon, USA). The following antibodies were used: antimouse CD16/32 'Fc block' and antimouse CD19-APC 'clone 1D3' (BD Biosciences), and antimouse CD8-FITC ‘clone KT15’ (ProImmune).

\section{Histopathological evaluation of the inflammatory response in} tumour tissue

Tumour specimens were collected and analysed as described in the online supplementary materials.

\section{Statistical methods}

All comparisons were performed using GraphPad Prism, Macintosh (V.5.0b, 2003; GraphPad Software, San Diego, California, USA) and Microsoft Excel 2011, Macintosh (V.14.3.9; Microsoft, Redmond, Washington, USA). Kinetic measurements were compared using the area under the curve (Excel). Parametrical data were compared using the analysis of variance or Student's t-test, and non-parametrical data with the MannWhitney U test.

\section{RESULTS}

\section{HCV replicon cells maintain viral antigen expression in the} absence of selection

We aimed at developing a mouse model based on the syngeneic transplantation of Hep56.1D (C57BL/6J-derived) hepatoma cells containing a genotype 2 a subgenomic replicon as described previously ${ }^{17}$ (figure $1 \mathrm{~A}$ ). In the initial set of experiments, two replicon cell clones were selected, designated 21-3 and $21-10^{17}$ supporting HCV RNA replication to a level comparable with the one in cells of the well-established human hepatoma cell line Huh7 (subclone Lunet; figure 1B). This was well reflected in similar expression levels of viral proteins as revealed by NS5A-specific western blot (figure 1C) and comparable subcellular localisation of viral proteins as exemplified for NS3 (figure 1D). Thus, the murine replicon cells are representative of the human replicon cells.

As G418 selection for HCV replicons is not possible in vivo, we determined maintenance of HCV replicon RNA and antigen expression in the cells in the absence of G418. As shown in figure $1 \mathrm{E}$, in spite of some fluctuation, $\mathrm{HCV}$ replicon cells retained NS3 protein expression at least up to 7 weeks in the absence of G418 selection, similar to what we had found earlier for human replicon cells. ${ }^{30}$ Of note, Hep56.1D cells stably expressing NS3/4A protein (NS3/4A hepatoma) from an integrated DNA copy and used as control had NS3 protein expression 
levels comparable with those in the $\mathrm{HCV}$ replicon clones (data not shown).

\section{HCV replicon cells form solid tumours in vivo}

To determine the ability of HCV replicon clones to form solid tumours, we injected 5 million cells subcutaneously into the right flank of C57BL/6J mice and measured tumour growth using a sliding calliper (figure 1F). Both replicon clones, as well as the stably transfected NS3/4A hepatoma cells (data not shown), formed palpable solid tumours at the site of inoculation with tumour sizes peaking between 8 and 16 days postinoculation (figure $1 \mathrm{~F}$ and data not shown). The Hep56.1D and HCV replicon clones 21-3 (figure $1 \mathrm{~F}$ ) and 21-10 (data not shown) as well as the stably transfected NS3/4A hepatoma cells (data not shown) all had similar in vivo growth kinetics. Interestingly, the HCV replicon clone 21-3 primed a weak HCV-specific T cell response by day 24 postinoculation (figure $1 \mathrm{~F}$ ), whereas the Hep56.1D-derived replicon cell clone 21-10 and the parental Hep56.1D cells did not (data not shown and figure 1F, respectively). This suggested that antigen production, and thus HCV RNA replication, was maintained in vivo in the HCV replicon cell clone 21-3, which therefore was chosen for further studies and is designated the 'HCV replicon cell line' in the following. To allow for non-invasive in vivo imaging of the tumour and to normalise for the number of tumour cells present at each time point, this HCV replicon cell line was stably transfected with a firefly luciferase (Fluc) gene (figure $1 G$ ) and was found to have integrated two luciferase gene copies/cell.

\section{Characterisation of HCV replicon cell tumours}

To determine the impact of an HCV-specific immune response on tumour cell growth, mice were either immunised with an HCV NS3/4A DNA vaccine or unrelated DNA once or twice (4 weeks apart), or left unimmunised, and challenged with the Hep56 tumour cells 2 weeks later (figure 1G). Solid Hep56.1D-derived tumours formed in vivo were characterised with respect to volume, HCV RNA and Fluc DNA copy numbers, as well as histological appearance, including H\&E staining, specific staining for Troma-1/cytokeratin 8 (CK8: to detect hepatoma cells) and CD3 (to detect T/NKT cells). These parameters were determined both in naïve mice and in mice vaccinated with a HCV NS3/4A DNA 2 weeks prior to tumour cell challenge. The tumour volume as determined by a sliding calliper peaked around days 8-16 post challenge, depending on the experiment (figure $1 \mathrm{~F}$ and online supplementary figure 1 ).

HCV RNA could be detected in tumours by both in situ hybridisation (figure 2A) and by RT-qPCR (figure 2B,C) indicating $\mathrm{HCV}$ RNA persistence in vivo. HCV replicon cell tumours grew much stronger in control (OVA and naïve) mice lacking an $\mathrm{HCV}$-specific $\mathrm{T}$ cell response compared with vaccinated animals as determined by both caliper and Luc DNA copy numbers (figure 2B, left and middle panels). However, levels of HCV RNA declined rather slowly throughout the observation period, indicating persistence of HCV RNA or fragments thereof in non-replicon cells (figure 2B). To verify the possible early effect of T cells on HCV RNA levels, the data from two independent experiments (figure $2 \mathrm{~B}, \mathrm{D}$ ) at day 4 were pooled and analysed statistically. Lower HCV RNA levels were observed in HCV NS3/4A-DNA immunised mice, as compared with control animals lacking HCV-specific T cells (figure 2C), arguing for immune control of HCV replicon cells.

To further corroborate these results, HCV RNA kinetics were determined in naïve and NS3/4A DNA vaccinated wild-type
$(\mathrm{CD} 4+/ \mathrm{CD} 8+)$, CD4-deficient (CD4-/CD8+) and CD8-deficient $(\mathrm{CD} 4+/ \mathrm{CD} 8-)$ mice challenged with the HCV replicon cells (figure 2D). As groups consisted of only three mice, we added the same groups from days 4 and 8 , or 8 and 12, or 4, 8 and 12 to allow for statistical calculations. We found that mice with NS3/4A-specific T cells had lower HCV RNA levels than naïve mice for all analysed groups $(\mathrm{p}<0.05$, Mann-Whitney). Thus, in spite of profound variability of HCV RNA levels, T cells seem to have an early inhibitory effect on HCV RNA replication in this model. Of note, both CD4+ and CD8 $+\mathrm{T}$ cells were essential for protection against HCV replicon tumour growth (online supplementary figure 2).

In order to confirm the role of $\mathrm{T}$ cells in control of $\mathrm{HCV}$ replicon cells (gt $2 \mathrm{a}$ ), we challenged NS3/4A DNA vaccinated $\mathrm{NS} 3 / 4 \mathrm{~A}$ (gt1a)-Tg mice that owing to tolerance have a gt1a-specific dysfunctional $\mathrm{T}$ cell response to this HCV antigen. The $\mathrm{HCV}$ replicon tumours were smaller at day 4 in the presence of HCV-specific T cells in both wild-type and NS3/4A(gt1a)-Tg mice (figure $3 \mathrm{~A}, \mathrm{~B}$ ). Later the protection against $\mathrm{HCV}$ replicon tumours was dependent on a functional genotype-specific $\mathrm{T}$ cell response that appeared to be lacking in NS3/4A(gt1a)-Tg mice (figure 3).

In conclusion, HCV replicon cells effectively establish tumours with HCV RNA replication persisting for at least 2-3 weeks in different syngeneic mouse strains. Our results suggest a very transient $\mathrm{T}$ cell-mediated control of $\mathrm{HCV}$ replication at early time points, which is lost at later time points. Yet protection against HCV replicon tumour cell growth is clearly mediated by functional genotype-specific $\mathrm{T}$ cells.

\section{A massive inflammatory response in the absence of early HCV-specific T cells}

The drivers for tumour growth in the non-vaccinated mice were both the expansion of Troma-1/CK-8-positive HCV replicon cells and the inflammatory response to the tumour (figure 4). Importantly, simultaneous with the expansion of HCV replicon cells at days 4 and 8 (figure 4) with HCV RNA replication (figure 2A,B), we observed a massive polyclonal inflammatory infiltrate containing $\mathrm{CD}^{+}$cells (data not shown), granulocytes and macrophages (figure 4). Thus, in this mouse model HCV RNA replication coexists with a strong inflammatory response, similar to what is a hallmark for HCV infection of humans.

The infiltrate, including $\mathrm{CD}^{+}$cells, was more pronounced in non-vaccinated mice, suggesting that uncontrolled growth of HCV replicon cells, or NS3/4A-expressing hepatoma cells, attracted a non-specific inflammatory response (figure 4). In contrast, when tumour growth was controlled by vaccine-induced HCV-specific T cell response, as in the vaccinated mice, the inflammatory response was much reduced (figure 4). Such a negative correlation between CTL function and an unspecific inflammatory infiltrate has been observed in human livers of persistently HBV-infected individuals that were unable to control viral replication. ${ }^{31}$ Thus, in this presented mouse model, an early HCV-specific T cell response has transient effects on HCV RNA replication, but can prevent a strong inflammatory response and expansion of HCV replicon cells in vivo.

\section{Characterisation of a T cell response that protects against HCV replicon cell tumours}

We found that an optimal priming of HCV-specific $\mathrm{T}$ cells is necessary for protection against growth of HCV replicon cells. This priming required DNA vaccination via in vivo electroporation (EP) to generate a response that protected against tumour 
a) HCV RNA detected by in situ hybridization

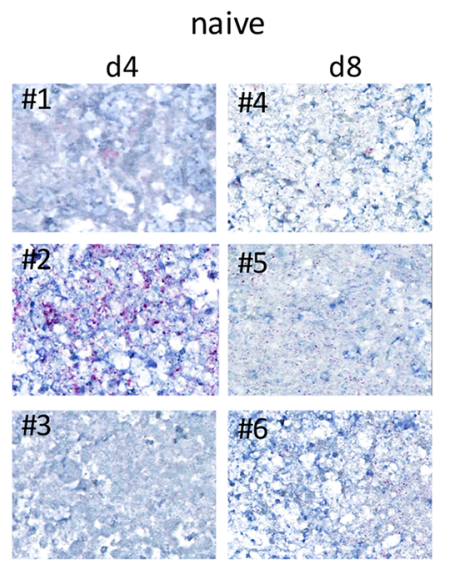

b) Kinetics of replicon cells at days 4-16

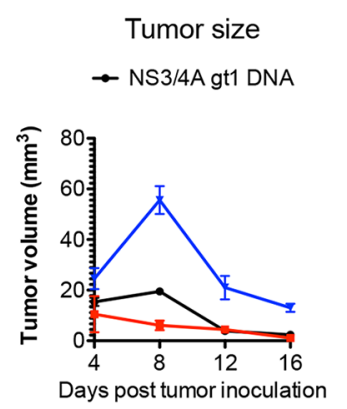

NS3/4A DNA gt2 vaccinated

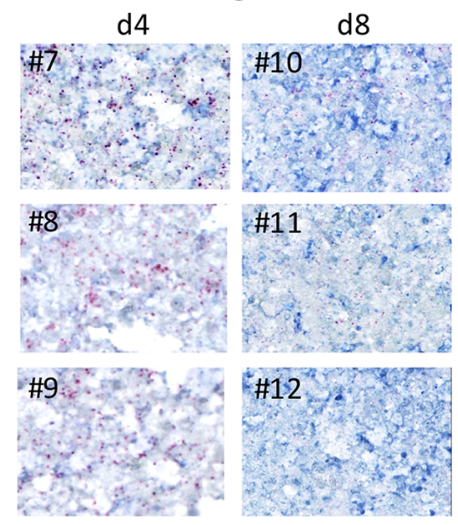

c) Mean HCV RNA at day 4 from two experiments

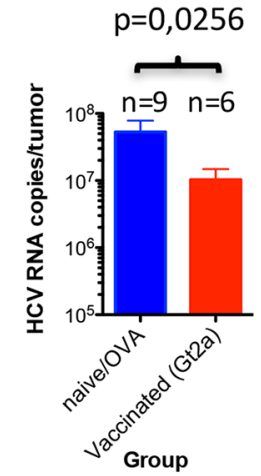

d) Role of NS3/4A gt2-specific CD4+ and CD8+ T cells in the early transient inhibition of HCV RNA levels
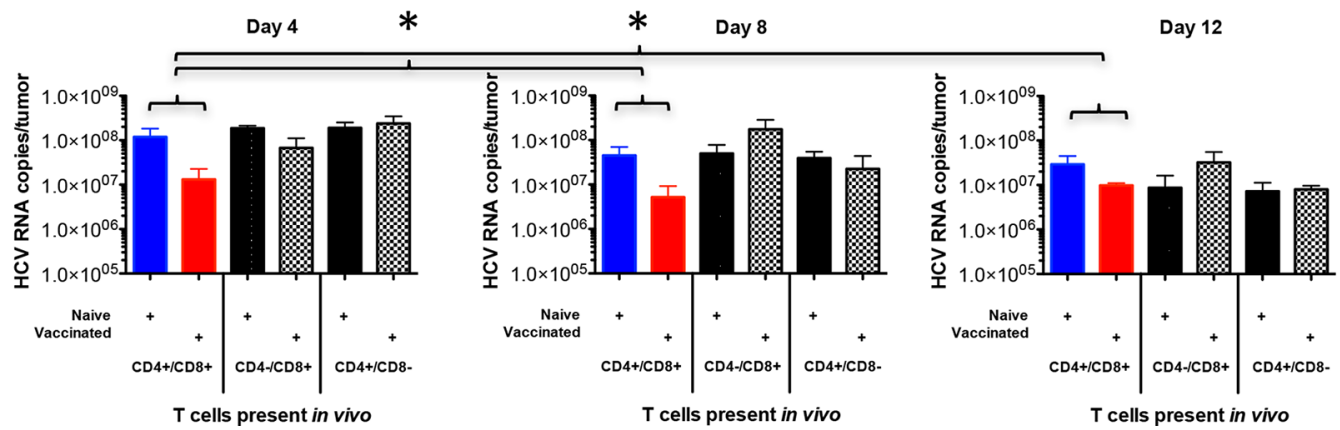

Figure 2 Detection of HCV RNA by chromogen in situ hybridisation and correlation of tumour growth, HCV RNA levels and effects of T cell immunity. (A) Detection of HCV RNA by chromogen in situ hybridisation (purple staining) in paraffin-embedded tumour tissues. Wild-type animals and mice immunised once with $50 \mu \mathrm{g}$ of HCV NS3/4A-gt2 DNA using in vivo EP were challenged with HCV replicon-expressing hepatoma cells, and HCV RNA was detected in individual mice at days 4 and 8 postinoculation by in situ hybridisation. (B) Side-by-side comparison of tumour size, number of luciferase (Luc) gene-positive cells and HCV RNA copies per tumour. The blue line represents both OVA-DNA immunised and non-immunised mice (naïve controls). The black line represents NS3/4A-gt1 a DNA (50 $\mu \mathrm{g}$ DNA with in vivo EP) immunised mice and the red line represents NS3/4Agt2a DNA ( $50 \mu \mathrm{g}$ DNA with in vivo EP) immunised mice. (C) Comparison of the HCV RNA copy number in tumours at day 4 from naïve or OVA DNA immunised mice (controls) and NS3/4A DNA immunised mice (50 $\mu \mathrm{g}$ DNA with in vivo EP) from two separate experiments. Statistical comparison with Mann-Whitney U-test. (D) Comparison of HCV RNA copy number per tumour in relation to HCV-specific T cell immunity at days 4, 8, and 12 posttumour challenge. Groups of wild-type (CD4+/CD8+), CD4-deficient (CD4-/CD8+) or CD8-deficient (CD4+/CD8-) mice were either immunised with NS3/4A-gt2a DNA ( $50 \mu \mathrm{g}$ DNA with in vivo EP) or left non-immunised or immunised with a control OVA-encoding DNA (OVA-DNA). All mice were challenged with the HCV replicon cells. $A^{\prime * \prime}$ sign indicates $p<0.05$ as determined by Mann-Whitney $\mathrm{U}$ test. When the values from the wild-type group at days 4 and 8 , or days 4,8 , and 12 , were added, there was a significant difference between the naïve and the vaccinated group with respect to HCV RNA levels ( $p<0.05$, respectively, Mann-Whitney). This was also true when the values from the wild-type group and CD4 deficient group at days 4 were added together ( $<<0.05$, respectively, Mann-Whitney; not indicated in graph). Different groups were added because each group consisted of three animals. EP, electroporation. 
a)

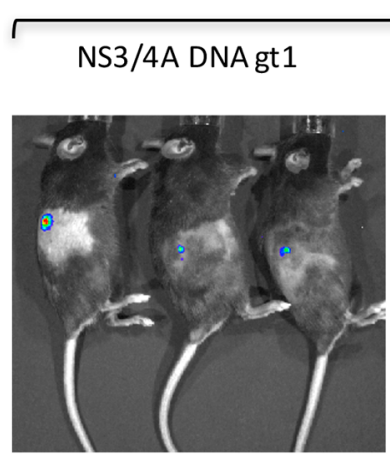

NS3/4A DNA gt2

OVA DNA
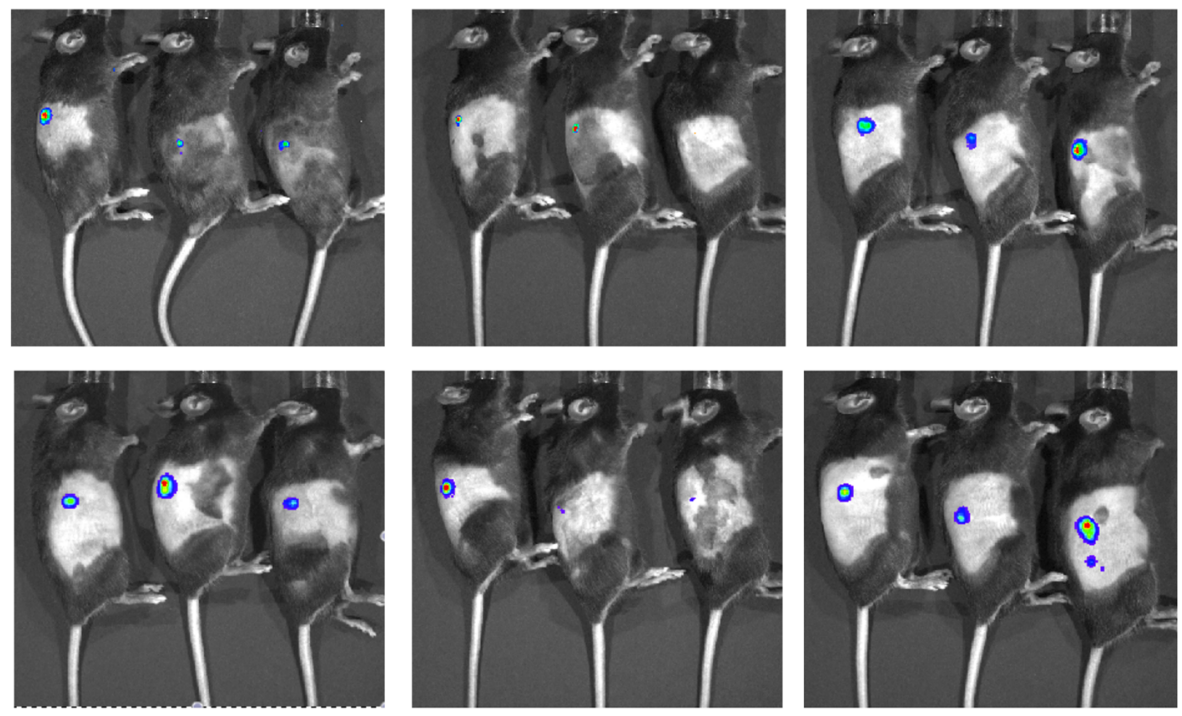

wild type
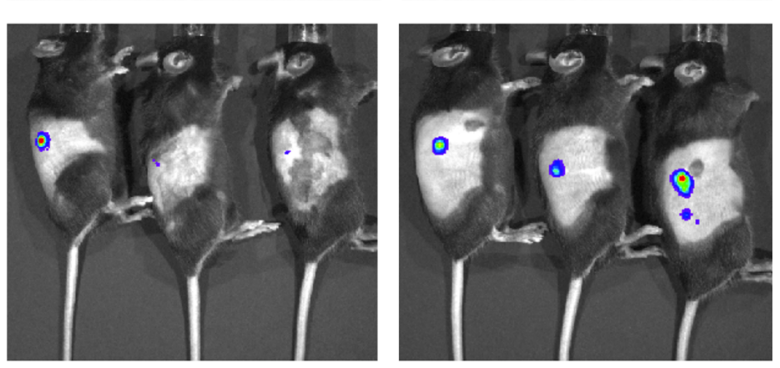

NS3/4A-Tg gt1

b)
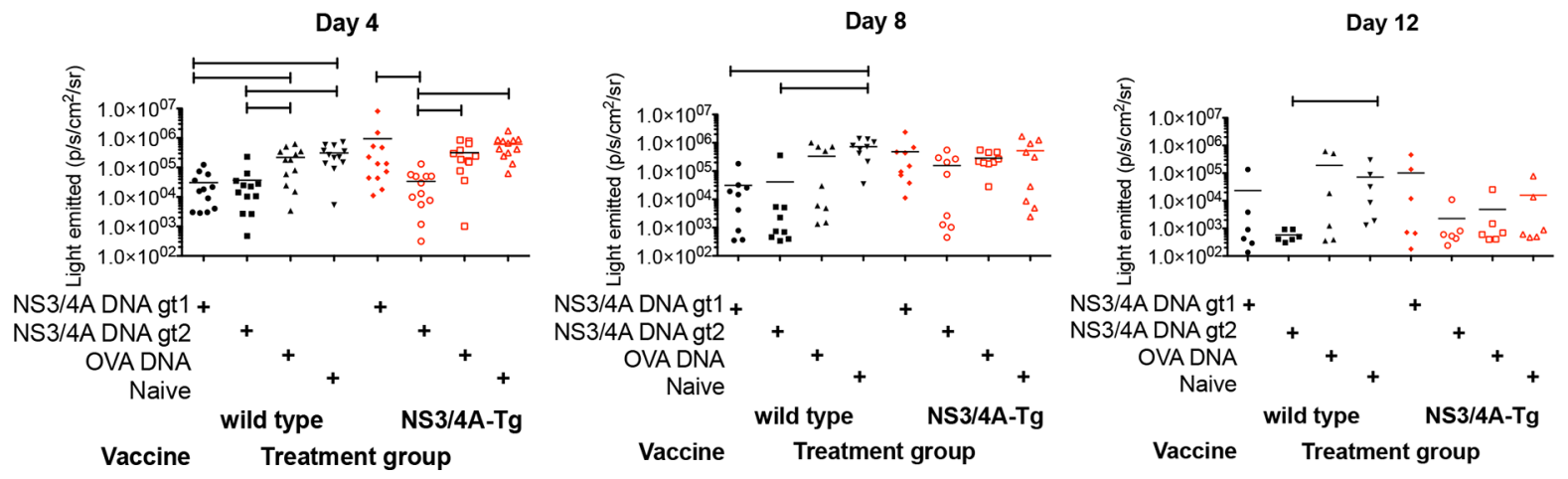

Figure 3 Relation between protection against tumour growth and HCV-specific DNA vaccination in wild-type and HCV NS3/4A-gt1a transgenic mice. Groups of 12 wild-type (wt) and HCV NS3/4A-gt1 transgenic (Tg) mice were either immunised with $50 \mu \mathrm{g}$ of NS3/4A-gt1 a DNA or NS3/4Agt2a DNA or left non-immunised or immunised with an irrelevant plasmid DNA (OVA-DNA). Two weeks after the last immunisation, all mice were challenged with the HCV replicon-containing hepatoma cell line 21/3 stably expressing in addition firefly luciferase. (A) The protection against tumour growth was analysed by measuring the biodistribution of firefly luciferase determined by in vivo imaging of immunised and non-immunised mice at days 4,8 and 12 post tumour cell inoculation. Representative in vivo imaging pictures are shown. (B) Summary of the imaging data that are given as the emitted light of individual mice $\left(\mathrm{p} / \mathrm{s} / \mathrm{cm}^{2} / \mathrm{sr}\right)$. The horizontal line shows the mean emitted light of each group (+SEM). * $\mathrm{p}<0.05$ as determined by the Mann-Whitney U test.

growth (online supplementary figure 2B). However, tumour growth was only partially controlled when using the same NS3/4A DNA dose, but administered intramuscularly without EP (online supplementary figure $2 \mathrm{C}$ ). It is worth noting that the (presumed) CD4 $+\mathrm{T}$ cells that were detected were cross-reactive between recombinant NS3/4A gt 1 and gt 2 antigens, suggesting a different quality of the early vaccine-primed CD4+ T cells as compared with those primed by the HCV replicon cell challenge (online supplementary figure $2 \mathrm{~A}$ vs figure $2 \mathrm{~B}, \mathrm{C}$ ).

To better understand the importance of the role of HCV RNA replication in the HCV replicon cell transplantation model, we used a Hep56.1D-derived NS3/4A cell line stably expressing gt2a NS3/4A as control. The NS3/4A protease complex has potent immune modulatory properties and therefore is a suitable control to determine effects that are not simply due to NS3/4A protein expression. To measure $\mathrm{T}$ cell responses, we first identified two $\mathrm{H}-2^{\mathrm{b}}$-restricted NS3/4A gt2-specific CTL epitopes (online supplementary figure 3 ). One of these epitopes was also suitable to quantify NS3/4A gt2-specific CTLs by using the DimerX technology (online supplementary figure 4). This also allowed us to study genetic variations (ie, immune escape) within the epitopes as they might emerge during replicon cell tumour growth.

Taking advantage of these novel tools, we monitored the kinetics of HCV NS3/4A epitope-specific and the global (peptide pools) $\mathrm{T}$ cell response to NS3/4A, and its role in protecting against the HCV replicon cell-induced tumours (figure 5). Groups of mice were vaccinated with NS3/4A gt2a DNA, or left 


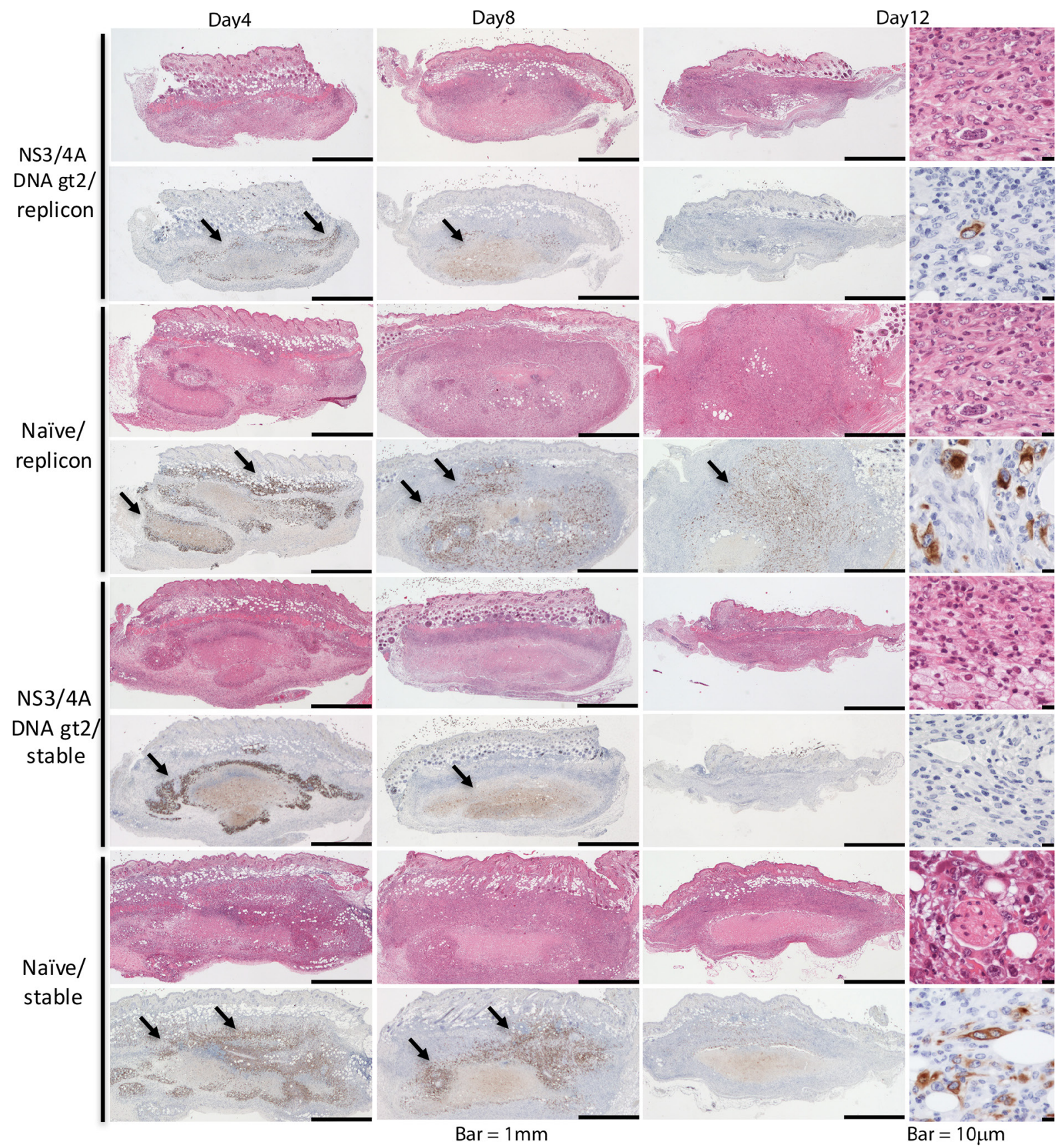

Figure 4 Histological analysis of tumour tissues from immunised and non-immunised mice. Histological analysis of tumour tissues from NS3/4Agt2a immunised (50 $\mathrm{gg}$ gt2 DNA, administered with in vivo electroporation) or non-immunised (naïve) wild-type mice challenged with HCV replicon cells or Hep56.1D cells stably expressing NS3/4A. Samples were taken at days 4, 8, and 12 postinoculation. Paraffin-embedded tumour sections were stained with H\&E to determine the inflammatory infiltrate (red panels), and specific staining of TROMA-I/cytokeratin 8 was performed to detect hepatoma cells (brown/blue panels). Representative histological pictures are shown.

untreated (naïve), and challenged with Hep56.1D-derived HCV replicon cells or stably NS3/4A-expressing cells 2 weeks later. Vaccination with the NS3/4A gt2a DNA protected mice against $\mathrm{HCV}$ replicon cell-induced tumours (figure $5 \mathrm{~A}$, blue line graphs), with the dominant response directed to the peptide pools (ie, both CD4+ and CD8+ T cells) peaking around days 8-12 (figure 5A; black bar graphs). This vaccine-induced pre-existing $\mathrm{T}$ cell response remained broad and seemed to be marginally boosted by the tumour cell challenge, but controlled HCV replicon cell tumour growth (figure 5A). In contrast, the non-vaccinated mice developed only a poor T cell response at days 12-16 that was unable to control tumour growth (figure 5A). Thus, an early IFN $\gamma$-producing CD4 + and CD8 + T cell response controls $\mathrm{HCV}$ replicon cell tumour growth. However, HCV-specific $\mathrm{CD} 4+$ and CD8 + T cells were only poorly boosted by the HCV replicon cell challenge (figure $5 \mathrm{~A}$ ). In addition, the weak $\mathrm{T}$ cell response primed by the $\mathrm{HCV}$ replicon cells alone was unable to control tumour growth (figure 5A).

A pre-existing $\mathrm{T}$ cell response also protected against growth of the stably NS3/4A-expressing hepatoma cells; however, with an impressive boosting effect dominated by CTLs (figure $5 \mathrm{~B}$ ). The $\mathrm{T}$ cell response narrowed over time to target only the NS3/4A peptide pools that included the CTL epitopes at 8-20 days after tumour challenge (figure $5 \mathrm{~B}$ ). Of note, this dominant CTL 
NS3/4A DNA

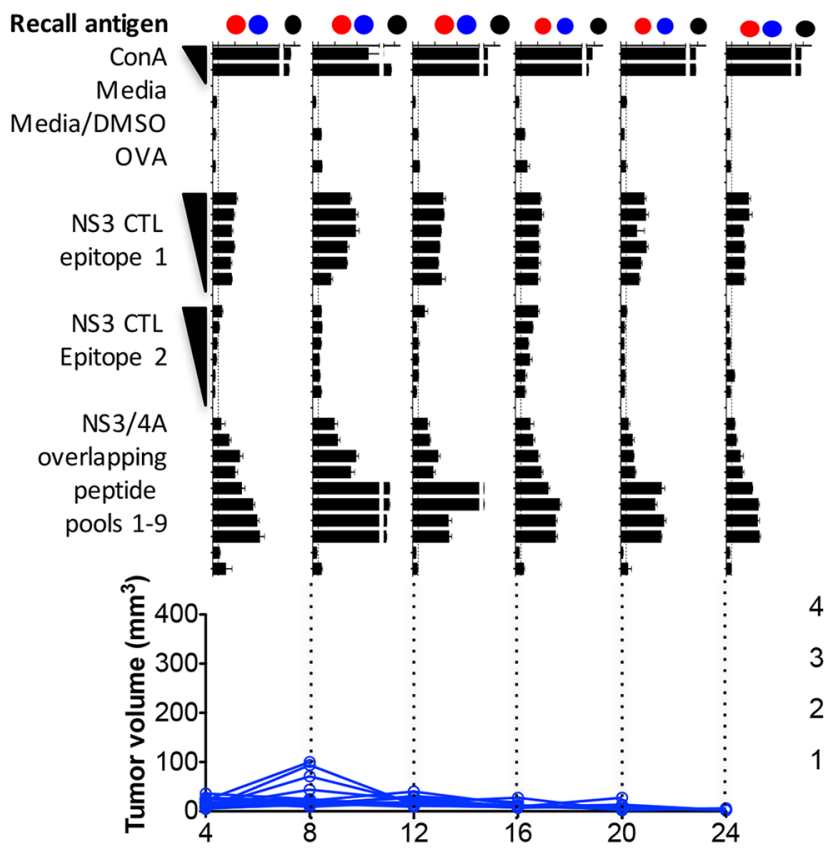

Naive

b) NS3/4A hepatoma

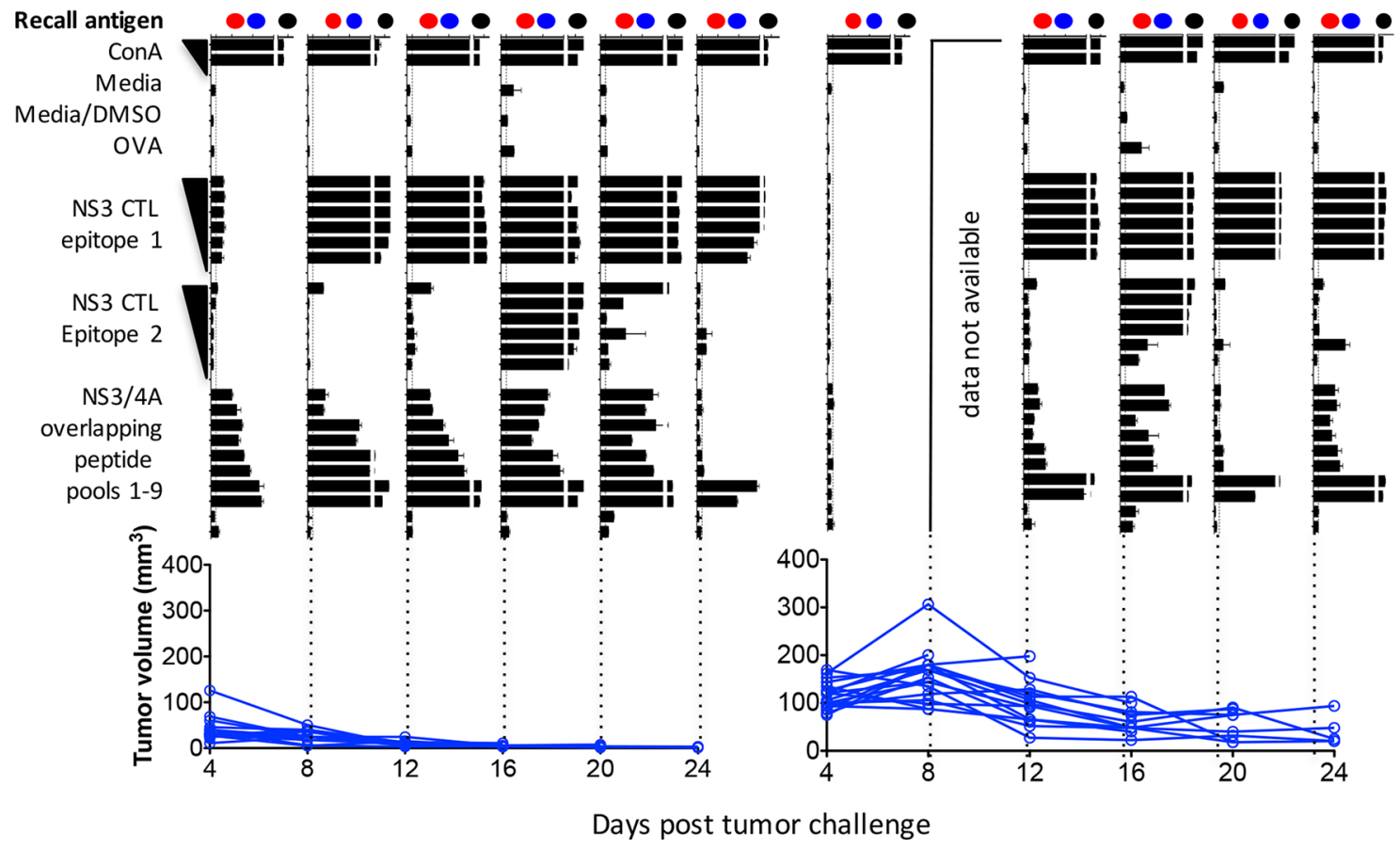

Figure 5 Relation between protection against tumour growth and HCV-specific T cell immunity. Kinetic (days 4, 8, 12, 16, 20 and 24) of tumour growth in groups (20 mice/group) of wild-type mice immunised twice with $50 \mu \mathrm{g}$ of NS3/4A-gt2a-DNA vaccine using in vivo electroporation (NS3/4A DNA; left panel) or non-immunised (naïve; right panel). All mice were given $5 \times 10^{6} \mathrm{HCV}$ replicon hepatoma cells (A) or the hepatoma cells stably expressing NS3/4A (B) subcutaneously into the right flank. Tumours were measured through the skin every second to third day using a sliding caliper. The tumour volume of individual mice is shown (blue lines). At indicated time points, mice were sacrificed and splenocytes harvested for determination of the number of IFN $\gamma$ spot-forming cells (SFCs) in wild-type mice by using ELISpot assay. The production of IFN $\gamma$ was determined after in vitro stimulation of splenocytes with de-escalating doses of the following antigens: two CTL peptides (NS3-CTL epitope 1 and epitope 2; 20, 2, 0.2, $0.02,0.002,0.0002 \mu \mathrm{g} / \mathrm{mL}$ ), and five NS3/4A overlapping peptide pools ( 15 peptides per pool, total concentration $7.5 \mu \mathrm{g} / \mathrm{mL}$ as indicated). As control antigens OVA-CTL $(1 \mu \mathrm{g} / \mathrm{mL})$ and ConA $(2$ or $1 \mu \mathrm{g} / \mathrm{mL})$ were used. Results are given as the mean SFCs $/ 10^{6}$ (+SD) splenocytes with a cut-off set at 50 SFCs $/ 10^{6}$ splenocytes. The red, blue and black dots on the y-axis indicate 400, 800 and 3000 IFN $\gamma$-producing SFC/10 ${ }^{6}$ cells, respectively. 
a

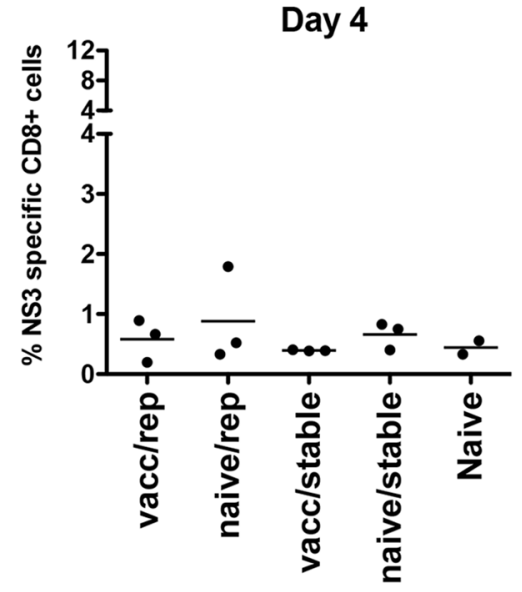

C

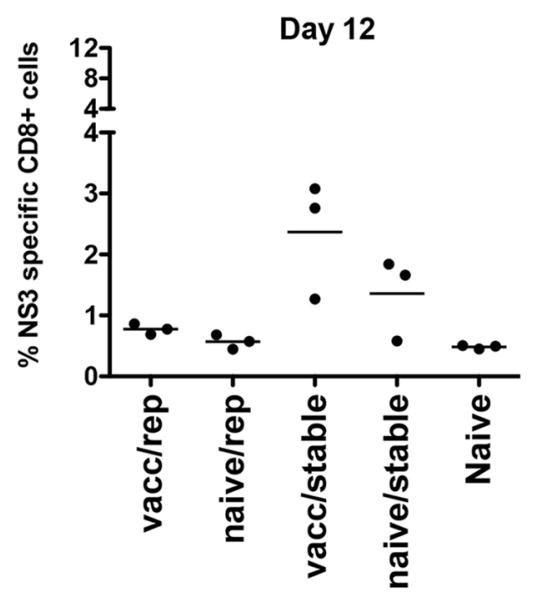

e

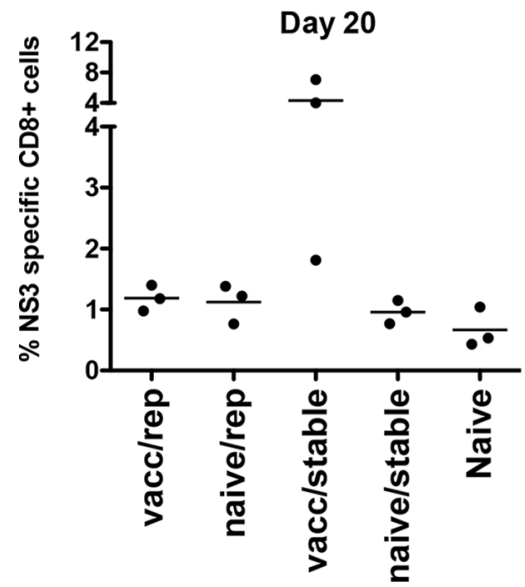

b

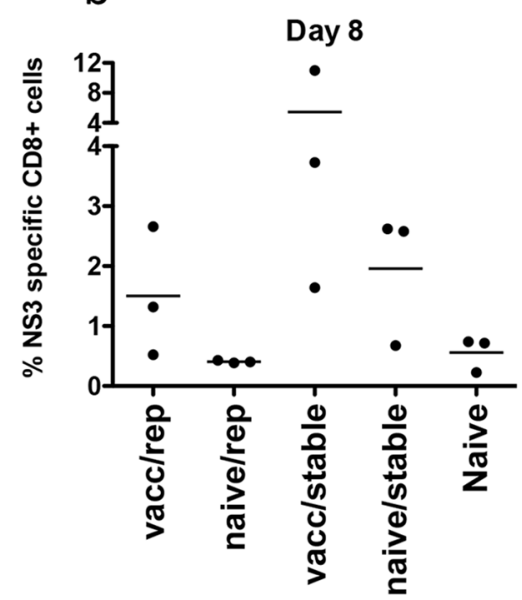

d

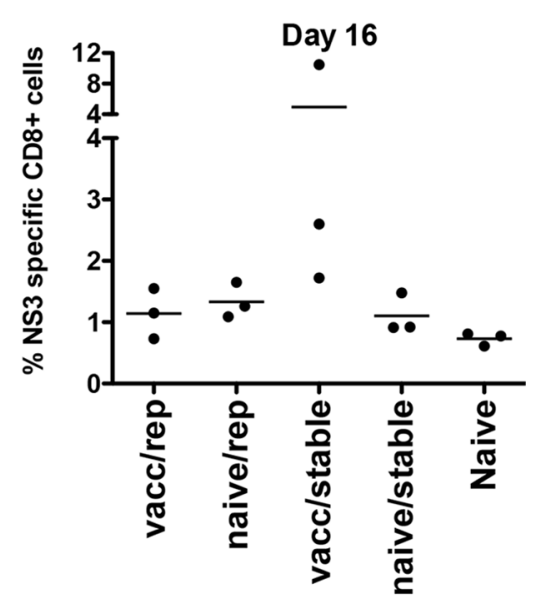

$f$

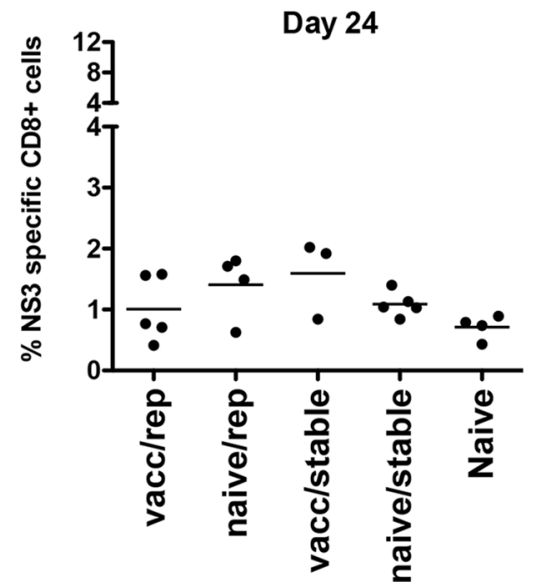

Figure 6 Quantification of the kinetics of NS3-specific CD8+ T cell responses in wild-type mice postimmunisation and tumour challenge. The expansion of NS3-specific CD8 ${ }^{+} \mathrm{T}$ cells in wild-type mice was determined using direct ex vivo staining of splenocytes with a recombinant soluble dimeric mouse $\mathrm{H}-2 \mathrm{D}(\mathrm{b}): \lg$ fusion protein preloaded with the NS3 CTL epitope APPPSWDAM (A-F). Groups of mice were immunised twice with $50 \mu \mathrm{g}$ of an NS3/4A-gt2a-DNA vaccine using in vivo electroporation or left non-immunised. All mice were challenged with $5 \times 10^{6} \mathrm{HCV}$ replicon hepatoma cells or NS3/4A-expressing hepatoma cells injected subcutaneously into the right flank. Quantification of APPPSWDAM epitope-specific CD8 ${ }^{+} T$ cells was performed at days 4, 8, 12, 16, 20 and 24 postinoculation. Each group consists of three to five mice per time point. APPPSWDAM epitopespecific $C D 8^{+} T$ cells are shown as the percentage of NS3-specific $C D 8^{+} T$ cells where each filled black circle represents an individual mouse. The black horizontal line indicates the mean of the group. Vacc, NS3/4A DNA immunised; naïve, non-immunised; rep, HCV replicon cells; stable, hepatoma cells stably expressing NS3/4A. 
epitope-directed response was distinct from that induced by the HCV replicon cells (figure 5A). Challenge with the NS3/4A hepatoma cells in the non-vaccinated group primed a strong and even more narrow response peaking at day 16 (possibly present at day 8) and lasting until at least day 24, and a weaker response to the peptide pools (figure $5 \mathrm{~B}$ ).

These differences were also reflected in the expansion of epitope-specific CTLs quantified by flow cytometry using the DimerX technology (figure 6). A challenge with HCV replicon cells barely recalled a detectable CTL expansion, whereas the NS3/4A hepatoma cells recalled a prominent CTL expansion at days 8-12 after replicon cell transplantation (figure 6). Thus, there are significant differences in the responses primed and boosted by the HCV replicon cells and the stably transfected NS3/4A hepatoma cells, despite expression of comparable levels of an enzymatically active NS3/4A protease. Hence, the weaker response primed by the HCV replicon cells supports the notion that HCV replication might attenuate the cellular immune response and that HCV RNA replication per se might exert immune regulatory effects.

\section{DISCUSSION}

There is a lack of simple and readily available small animal models to study the effect of HCV vaccines. Several models have been generated, but all have their individual drawbacks. ${ }^{9-11} 1632$ The most advanced model is the transgenic mouse allowing for $\mathrm{HCV}$ replication, but requiring a STAT1 ${ }^{-/-}$background, which severely impairs both the innate and the adaptive immune response. ${ }^{1632}$ This model is unfortunately not useful for studying in vivo primed $T$ cell responses, and in addition requires extensive backcrossing to study the role of individual host genes. Several transgenic lineages expressing all or selected HCV proteins have been generated and these are useful to study vaccine-induced $\mathrm{T}$ cell responses in the presence of a dysfunctional $\mathrm{T}$ cell response. ${ }^{9-11}$ However, these mouse models lack HCV RNA replication and the degree of $\mathrm{T}$ cell dysfunction varies greatly depending on the timing and levels of the transgene expression. Thus, there is a need for a simple model supporting HCV RNA replication.

We generated a mouse model based on a mouse hepatoma cell line supporting the replication of subgenomic HCV replicons. ${ }^{17}$ When these HCV replicon cells were injected subcutaneously into syngeneic mice, a solid tumour was formed in the absence of an early functional HCV-specific T cell response. Despite its limitations, many aspects of this model replicate what we know from the infection of humans. First, the HCV replicon cells, like the $\mathrm{HCV}$-infected liver, attract an influx of immune cells that are unable to control tumour growth. In the presence of an early and broadly specific CD4 + and $\mathrm{CD} 8+\mathrm{T}$ cell response, the expansion of HCV replicon cells and tumour formation is prevented, recapitulating the events in patients who spontaneously clear acute HCV infection. Second, injection of the HCV replicon cells failed to effectively prime a HCV-specific $\mathrm{T}$ cell response, contrasting the strong CTL-focused $\mathrm{T}$ cell response appearing after injection of hepatoma cells stably expressing an enzymatically functional HCV NS3/4A complex. In humans and chimpanzees a cleared HCV infection does not induce a protective immunity. ${ }^{33}$ Thus, much alike HCV infection of humans, the HCV replicon cells appear to be poorly immunogenic in vivo, possibly reflecting immune inhibitory effects of the HCV replication itself. ${ }^{34} 35$ Third, we do not yet know whether immune escape is one reason for the poor immunogenicity with respect to CTLs. In an attempt to address this question we sequenced parts of the replicon corresponding to two epitopic regions that were identified during the MHC I epitope screening (online supplementary figure 3 ). Although we could identify one synonymous change, this mutation also appeared in vitro after 12 days of culture without G418 (data not shown). Thus, we do not yet have evidence of genetic changes within these epitopic regions. However, genetic changes outside of the epitopic regions might be selected in vivo in the HCV replicon cell line. One observation might favour this assumption. Vaccinated wild-type mice challenged with the HCV replicon cells showed an increase in HCV RNA copy number per cell from days 4 to 16, arguing that a few cells might be selected with high levels of HCV RNA replication. This will be of importance to determine along with a detailed characterisation of the tumour infiltrating immune cells. Fourth, the HCV replicon cells can be rapidly applied to any genetic variant in the murine $\mathrm{H}-2^{\mathrm{b}}$ background for elucidation of the role of individual genes for immune control of HCV RNA replication and tumour formation.

Although the HCV replicon cell-based mouse system described here lacks many features of the HCV infection of humans such as release of infectious virus, difficulties in tracing HCV RNA replication and restriction to Hep56.1D hepatoma cells, its advantages outweigh these limitations. Of note, the $\mathrm{HCV}$ replicon cells mimic infection of humans in key points. Like in patients with chronic hepatitis $\mathrm{C}$, HCV replicon cells sustain self-replicating HCV RNAs in an inflammatory environment and are poorly immunogenic in vivo. Moreover, $\mathrm{HCV}$ replicon cells escape control in an immune-competent host with dysfunctional HCV-specific immunity. Finally, this immune-competent mouse model is highly versatile, because any $\mathrm{H}-2^{\mathrm{b}}$-restricted $\mathrm{C} 57 \mathrm{BL} / 6 \mathrm{~J}$-derived transgenic mouse lineage can be used for syngeneic transplantation. In all these respects, the system described here provides new opportunities to study the immune response to liver cells containing self-replicating HCV RNAs.

Author affiliations

'Department of Laboratory Medicine, Division of Clinical Microbiology, F68, Karolinska Institutet, Karolinska University Hospital Huddinge, S-141 86 Stockholm, Solna, Sweden

${ }^{2}$ Department of Infectious Diseases, Molecular Virology, University of Heidelberg, Heidelberg, Germany

${ }^{3}$ Division of Virus-Associated Carcinogenesis, German Cancer Research Center, Heidelberg, Germany

${ }^{4}$ Unit of Virus Assembly and Host, Key Laboratory of Molecular Virology \& Immunology, Institut Pasteur of Shanghai, Shanghai Institutes for Biological Sciences, Chinese Academy of Sciences, Shanghai, China

${ }^{5}$ German Center for Infection Research (DZIF) - Heidelberg Partner Site, Heidelberg, Germany

${ }^{6}$ Division of Virus-AssociatedCarcinogenesis, German Cancer Research Center, Heidelberg, germany

${ }^{7}$ Department of Laboratory Medicine, Division of Clinical Microbiology, Karolinska Institutet, Stockholm, Sweden

${ }^{8}$ Karolinska University Laboratory, Clinical Microbiology, Karolinska University Hospital, Stockholm, Sweden

Contributors RB and MS developed the original idea of the project. DR and GL established and characterised the HCV replicon cells and quantified HCV RNA levels in tumours. SL, FH, GA and LF performed all in vivo experiments, immunological and histological analysis. MS and RB obtained funding for the study. SL, FH, GA, LF, DR, $\mathrm{GL}, \mathrm{RB}$ and MS wrote the manuscript. All authors contributed to the final design of the study and reviewed the final manuscript.

Funding The study was supported by grants from the Swedish Science Council, the SwedishCancer Foundation, and the Stockholm County Council to MS. RB was supported bythe Deutsche Forschungsgemeinschaft (TRR179, TP9) and by a grant from theGerman Cancer Research Center.

Competing interests MS and LF are cofounders of Svenska Vaccinfabriken, which holds commercial rights to vaccine patents. RB is a cofounder of ReBLikoN GmbH, which holds commercial rights to HCV replicon technology. 
Patient consent No patients in the study.

Provenance and peer review Not commissioned; externally peer reviewed.

Open access This is an open access article distributed in accordance with the Creative Commons Attribution Non Commercial (CC BY-NC 4.0) license, which permits others to distribute, remix, adapt, build upon this work non-commercially, and license their derivative works on different terms, provided the original work is properly cited and the use is non-commercial. See: http://creativecommons.org/ licenses/by-nc/4.0/

(c) Article author(s) (or their employer(s) unless otherwise stated in the text of the article) 2018. All rights reserved. No commercial use is permitted unless otherwise expressly granted.

\section{REFERENCES}

1 Alvarez-Lajonchere L, Dueñas-Carrera S. Advances in DNA immunization against hepatitis C virus infection. Hum Vaccin 2009;5:568-71.

2 Alvarez-Lajonchere L, Shoukry NH, Grá B, et al. Immunogenicity of CIGB-230, a therapeutic DNA vaccine preparation, in HCV-chronically infected individuals in a Phase I clinical trial. J Viral Hepat 2009;16:156-67.

3 Barnes E, Folgori A, Capone S, et al. Novel adenovirus-based vaccines induce broad and sustained T cell responses to HCV in man. Sci Trans/ Med 2012;4:115ra1.

4 Weiland $\mathrm{O}$, Ahlén G, Diepolder $\mathrm{H}$, et al. Therapeutic DNA vaccination using in vivo electroporation followed by standard of care therapy in patients with genotype 1 chronic hepatitis C. Mol Ther 2013;21:1796-805.

5 Di Bisceglie AM, Janczweska-Kazek E, Habersetzer F, et al. Efficacy of immunotherapy with TG4040, peg-interferon, and ribavirin in a Phase 2 study of patients with chronic HCV infection. Gastroenterology 2014;147:119-31. e113.

6 Habersetzer $F$, Honnet G, Bain C, et al. A poxvirus vaccine is safe, induces T-cell responses, and decreases viral load in patients with chronic hepatitis $C$. Gastroenterology 2011;141:890-9. e891-894.

7 Swadling L, Capone S, Antrobus RD, et al. A human vaccine strategy based on chimpanzee adenoviral and MVA vectors that primes, boosts, and sustains functional HCV-specific T cell memory. Sci Trans/ Med 2014;6:261ra153.

8 Wedemeyer H, Schuller E, Schlaphoff V, et al. Therapeutic vaccine IC41 as late add-on to standard treatment in patients with chronic hepatitis $\mathrm{C}$. Vaccine 2009:27:5142-51.

9 Disson O, Haouzi D, Desagher S, et al. Impaired clearance of virus-infected hepatocytes in transgenic mice expressing the hepatitis C virus polyprotein. Gastroenterology 2004;126:859-72.

10 Frelin L, Brenndörfer ED, Ahlén G, et al. The hepatitis C virus and immune evasion: non-structural $3 / 4 \mathrm{~A}$ transgenic mice are resistant to lethal tumour necrosis factor alpha mediated liver disease. Gut 2006;55:1475-83.

11 Kriegs M, Bürckstümmer T, Himmelsbach K, et al. The hepatitis C virus non-structural NS5A protein impairs both the innate and adaptive hepatic immune response in vivo. J Biol Chem 2009;284:28343-51.

12 Majumder M, Ghosh AK, Steele R, et al. Hepatitis C virus NS5A protein impairs TNF-mediated hepatic apoptosis, but not by an anti-FAS antibody, in transgenic mice. Virology 2002;294:94-105.

13 Moriya $\mathrm{K}$, Fujie $\mathrm{H}$, Shintani Y, et al. The core protein of hepatitis $\mathrm{C}$ virus induces hepatocellular carcinoma in transgenic mice. Nat Med 1998;4:1065-7.

14 Moriya K, Yotsuyanagi H, Shintani Y, et al. Hepatitis C virus core protein induces hepatic steatosis in transgenic mice. J Gen Virol 1997;78:1527-31.
15 Pasquinelli C, Shoenberger JM, Chung J, et al. Hepatitis C virus core and E2 protein expression in transgenic mice. Hepatology 1997;25:719-27.

16 Dorner M, Horwitz JA, Donovan BM, et al. Completion of the entire hepatitis $C$ virus life cycle in genetically humanized mice. Nature 2013;501:237-41.

17 Long G, Hiet MS, Windisch MP, et al. Mouse hepatic cells support assembly of infectious hepatitis C virus particles. Gastroenterology 2011;141:1057-66.

18 Kress S, Jahn UR, Buchmann A, et al. p53 mutations in human hepatocellular carcinomas from Germany. Cancer Res 1992;52:3220-3.

19 Ahlén G, Derk E, Weiland M, et al. Cleavage of the IPS-1/Cardif/MAVS/VISA does not inhibit T cell-mediated elimination of hepatitis C virus non-structural 3/4A-expressing hepatocytes. Gut 2009;58:560-9.

20 Levander S, Sällberg M, Ahlén G, et al. A non-human hepadnaviral adjuvant for hepatitis C virus-based genetic vaccines. Vaccine 2016;34:2821-33.

21 Frelin L, Ahlén G, Alheim M, et al. Codon optimization and mRNA amplification effectively enhances the immunogenicity of the hepatitis C virus nonstructural 3/4A gene. Gene Ther 2004;11:522-33.

22 Boyle JS, Koniaras C, Lew AM. Influence of cellular location of expressed antigen on the efficacy of DNA vaccination: cytotoxic T lymphocyte and antibody responses are suboptimal when antigen is cytoplasmic after intramuscular DNA immunization. Int Immunol 1997;9:1897-906.

23 Lazdina U, Alheim M, Nyström J, et al. Priming of cytotoxic T cell responses to exogenous hepatitis B virus core antigen is B cell dependent. J Gen Virol 2003;84:139-46.

24 Frelin L, Brenndörfer ED, Ahlén G, et al. The hepatitis C virus and immune evasion: non-structural 3/4A transgenic mice are resistant to lethal tumour necrosis factor alpha mediated liver disease. Gut 2006:55:1475-83.

25 Ahlén G, Söderholm J, Tjelle T, et al. In vivo electroporation enhances the immunogenicity of hepatitis C virus nonstructural 3/4A DNA by increased local DNA uptake, protein expression, inflammation, and infiltration of CD3+ T cells. J Immunol 2007;179:4741-53.

26 Ahlén G, Frelin L, Holmström F, et al. A targeted controlled force injection of genetic material in vivo. Mol Ther Methods Clin Dev 2016;5:16016.

27 Jensen MM, Jørgensen JT, Binderup T, et al. Tumor volume in subcutaneous mouse xenografts measured by microCT is more accurate and reproducible than determined by 18F-FDG-microPET or external caliper. BMC Med Imaging 2008;8:16.

28 Ahlén G, Frelin L. Methods to evaluate novel hepatitis C virus vaccines. Methods Mol Biol 2016;1403:221-44.

29 Frelin L, Alheim M, Chen A, et al. Low dose and gene gun immunization with a hepatitis C virus nonstructural (NS) 3 DNA-based vaccine containing NS4A inhibit NS3/4A-expressing tumors in vivo. Gene Ther 2003;10:686-99.

30 Pietschmann T, Lohmann V, Rutter G, et al. Characterization of cell lines carrying selfreplicating hepatitis C virus RNAs. J Virol 2001;75:1252-64.

31 Bertoletti A, Gehring AJ. Immune therapeutic strategies in chronic hepatitis B virus infection: virus or inflammation control? PLoS Pathog 2013:9:e1003784.

32 Dorner M, Horwitz JA, Robbins JB, et al. A genetically humanized mouse model for hepatitis $C$ virus infection. Nature 2011;474:208-11.

33 Callendret B, Eccleston HB, Hall S, et al. T-cell immunity and hepatitis C virus reinfection after cure of chronic hepatitis $C$ with an interferon-free antiviral regimen in a chimpanzee. Hepatology 2014;60:1531-40.

34 Chen M, Sällberg M, Sönnerborg A, et al. Limited humoral immunity in hepatitis C virus infection. Gastroenterology 1999;116:135-43.

35 Martin B, Hennecke N, Lohmann V, et al. Restoration of HCV-specific CD8+ T cell function by interferon-free therapy. J Hepatol 2014;61:538-43. 